\title{
Increased rhythmicity in hypertensive arterial smooth muscle is linked to transient receptor potential canonical channels
}

\author{
Xiaoping Chen ${ }^{a}$, \#, Dachun Yang ${ }^{a}$, \#, Shuangtao Ma ${ }^{a}$, Hongbo He ${ }^{a}$, Zhidan Luo ${ }^{a}$, Xiaoli Feng ${ }^{a}$, \\ Tingbing Cao ${ }^{a}$, Liqun Ma ${ }^{a}$, Zhencheng Yan ${ }^{a}$, Daoyan Liu ${ }^{a, *}$, Martin Tepel ${ }^{\text {b }}$, Zhiming Zhu ${ }^{a}$ *' \\ ${ }^{a}$ Center for Hypertension and Metabolic Diseases, Department of Hypertension and Endocrinology, Daping Hospital, Third Military \\ Medical University, Chongqing Institute of Hypertension, Chongqing, China \\ ${ }^{b}$ Med. Klinik Nephrologie, Charite Campus Benjamin Franklin, Berlin, Germany
}

Received: June 8, 2009; Accepted: August 13, 2009

\begin{abstract}
Vasomotion describes oscillations of arterial vascular tone due to synchronized changes of intracellular calcium concentrations. Since increased calcium influx into vascular smooth muscle cells from spontaneously hypertensive rats (SHR) has been associated with variances of transient receptor potential canonical (TRPC) channels, in the present study we tested the hypothesis that increased vasomotion in hypertension is directly linked to increased TRPC expression. Using a small vessel myograph we observed significantly increased norepinephrine-induced vasomotion in mesenteric arterioles from SHR compared to normotensive Wistar-Kyoto (WKY) rats. Using immunoblottings we obtained significantly increased expression of TRPC1, TRPC3 and TRPC5 in mesenteric arterioles from SHR compared to WKY, whereas TRPC4 and TRPC6 showed no differences. Norepinephrine-induced vasomotion from SHR was significantly reduced in the presence of verapamil, SKF96365, 2-aminoethoxydiphenylborane (2-APB) or gadolinium. Pre-incubation of mesenteric arterioles with anti-TRPC1 and anti-TRPC3 antibodies significantly reduced norepinephrine-induced vasomotion and calcium influx. Control experiments with pre-incubation of TRPC antibodies plus their respective antigenic peptide or in the presence of anti- $\beta$-actin antibodies or random immunoglobulins not related to TRPC channels showed no inhibitory effects of norepinephrine-induced vasomotion and calcium influx. Administration of candesartan or telmisartan, but not amlodipine to SHR for 16 weeks significantly reduced either the expression of TRPC1, TRPC3 and TRPC5 as well as norepinephrine-induced vasomotion in mesenteric arterioles. In conclusion we gave experimental evidence that the increased TRPC1, TRPC3 and TRPC5 expression in mesenteric arterioles from SHR causes increased vasomotion in hypertension.
\end{abstract}

Keywords: vasomotion • transient receptor potential channels • angiotensin II 1 receptor blocker $\bullet$ calcium channel blocker $\bullet$ hypertension

\section{Introduction}

Vasomotion is the regular variation in tone of arteries. Vasomotion, e.g. oscillations of vascular tone due to synchronized oscillations of smooth muscle cell tension, occurs in arteries either spontaneously or in response to pressure, stretch or application of vasoconstrictor agonists [1-3]. Vasomotion is known to be associated with slow oscillations of smooth muscle membrane potential and of intracellular calcium concentrations. Vasomotion

\footnotetext{
\#These authors contributed equally.

*Correspondence to: Dr. Zhiming ZHU,

Department of Hypertension and Endocrinology,

Daping Hospital, Third Military Medical University,

Chongqing, PR China.

Tel.: +86-23-68767849

Fax: +86-23-68767847

E-mail: zhuzm@yahoo.com
}

may also be enhanced by oscillations of transplasmamembrane calcium influx [4-6]. In several experimental models of hypertension an increased vasomotion of arteries had been described. Lefer et al. observed enhanced vasomotion of cerebral arterioles in spontaneously hypertensive rats (SHR) compared to normotensive rats [7]. An increased vasomotion was observed in segments of posterior cerebral arteries and in mesenteric arteries from 
spontaneously hypertensive stroke-prone rats compared to Wistar-Kyoto (WKY) rats [8, 9].

Transient receptor potential canonical (TRPC) cation channels have been described to play an important role for transplasmamembrane calcium influx [10]. Recent experimental data from our group and other groups indicated that an increased TRPC expression is associated with hypertension. Liu et al. reported increased TRPC3 expression in several tissues from SHR compared to normotensive WKY rats $[11,12]$. Dietrich et al. showed that elevated TRPC3 channel expression in TRPC6 knockout mice was associated with increased vasoconstriction and increased blood pressure [13].

In the present study we therefore tested the hypothesis whether increased vasomotion in hypertension is associated with increased TRPC expression in mesenteric arterioles from SHR. Our study for the first time gives several experimental evidence that TRPC channels are responsible for increased oscillations of vascular tone in hypertension.

\section{Materials and methods}

The investigation conforms to the Guide for the Care and Use of Laboratory Animals published by the US National Institutes of Health (NIH Publications No. 85-23, revised 1996) and was approved by the local animal protection authorities.

\section{Animals and treatment}

In this study, 3-month-old SHR and WKY rats weighing 250-260 g were used. SHR were randomly divided into four groups: control group received placebo $(n=6), 5 \mathrm{mg} / \mathrm{kg} /$ day telmisartan $(n=6), 4 \mathrm{mg} / \mathrm{kg} /$ day candesar$\tan (n=6)$ and $10 \mathrm{mg} / \mathrm{kg} /$ day amlodipine $(n=6)$ by oral garage for 16 weeks. WKY were used as reference controls $(n=6)$ but were not treated with telmisartan, candisartan or amlodipine by oral garage. The rats were housed under a 12-hr/12-hr day/night cycle and given tap water and standard chow ad libitum. Systolic blood pressure was measured monthly in conscious and restrained rats by the tail-cuff method.

\section{Preparation of mesenteric arterioles and measurements of isometric tension}

At the end of treatment, rats were decapitated. The small intestine and associated mesenteric vessels were removed and placed in ice-cold oxygenated $\left(95 \% \mathrm{O}_{2}, 5 \% \mathrm{CO}_{2}\right)$ physiological salt solution (PSS) of the following composition (in $\mathrm{mmol} / \mathrm{l}$ ): $\mathrm{NaCl} 119.0, \mathrm{KCl} 4.7, \mathrm{KH}_{2} \mathrm{PO}_{4} \quad 0.4, \mathrm{NaHCO}_{3}$ 25.0, $\mathrm{MgSO}_{4}$ 1.17, $\mathrm{CaCl}_{2} 2.5$ and glucose 5.5. In $\mathrm{Ca}^{2+}$-free PSS, $\mathrm{Ca}^{2+}$ was omitted and $1 \mathrm{mmol} / \mathrm{l}$ EGTA was added. All solutions contained $2.5 \mathrm{mmol} / \mathrm{l}$ extracellular calcium if not indicated otherwise. Some experiments were performed in the absence of extracellular calcium to rule out the effects of transplasmamembrane calcium influx. Background fluorescence was determined after quenching extracellular fluorescent dye using manganese. For fluorescence measurements the vessels were mounted and stretch in the same way as in the contraction experiments. Second-order mesenteric arterioles were isolated for the study by careful removal the surrounding fat tissue; each artery was cut into cylindrical segments of $2.5 \mathrm{~mm}$ in length and the internal diameter of the vessels used in this study ranged between 100 and $150 \mu \mathrm{m}$. All the experiments were carried out on vessels with intact endothelium.

According to the method described by Huang et al. and Zhu et al. $[14,15]$, arterial rings were mounted in a multi myograph system (Danish Myo Technology A/S, Aarhus, Denmark) and changes of vessel tension were recorded by Power lab data recording system (AD Instrument, Castle Hill, Australia). Each arterial ring was bathed in PSS solution with $95 \% \mathrm{O}_{2}$ plus $5 \% \mathrm{CO}_{2}$ at $37^{\circ} \mathrm{C}, \mathrm{pH} 7.40$. The PSS solution was changed every $20 \mathrm{~min}$. The arterial rings were placed under an optimal resting tension, which was the minimum level of stretch giving the largest force after administration of $60 \mathrm{mmol} / \mathrm{K} \mathrm{KCl}$. The rings were allowed to stabilize at optimal resting tension for $90 \mathrm{~min}$. before the start of the experiments.

Vasomotion of mesenteric arterioles was induced by norepinephrine (final concentration, $10 \mu \mathrm{mol} / \mathrm{l}$ ) in the absence and presence of TRP channel blockers, 1-[2-(4-Methoxyphenyl)-2-[3-(4-methoxyphenyl)propoxy]et hyl-1H-imidazole hydrochloride (SKF96365, final concentration, $10 \mu \mathrm{mol} / \mathrm{l})$, of 2-aminoethoxydiphenylborane (2-APB, final concentration, $10 \mu \mathrm{mol} / \mathrm{l}$ ) and gadolinium (final concentration, $100 \mu \mathrm{mol} / \mathrm{l}$ ). According to established techniques the magnitude of vasomotion was quantified as the rhythmic oscillatory waves, expressed as the percentage of the vasoconstriction produced by $60 \mathrm{mmol} / \mathrm{KCl}[7,8]$. Additional experiments were performed after calcium depletion of sarcoendoplasmic reticulum by thapsigargin (final concentration $1 \mu \mathrm{mol} / \mathrm{l}$ ) for $20 \mathrm{~min}$. in the absence of extracellular calcium.

Vasomotion was also investigated in mesenteric arterioles after preincubation with antibodies against TRPC or against $\beta$-actin (1:200) for $40 \mathrm{~min}$. at $37^{\circ} \mathrm{C}$. Polyclonal anti-TRPC1, anti-TRPC3 and anti-TRPC5 antibodies generated in rabbits were purchased from Alomone Labs (Jerusalem, Israel) or Sigma-Aldrich (St. Louis, MO, USA). The selectivity and negligible cross-reactivity of these anti-TRPC antibodies for their target proteins have been previously described $[15,16]$. Control experiments were conducted in mesenteric arterioles incubated in PSS with immglobulins (Sigma, $3 \mu \mathrm{g} / \mathrm{ml}$ ) or albumin (Sigma, $3 \mu \mathrm{g} / \mathrm{ml}$ ) or in the presence of a mixture of anti-TRPCs antibodies and respective antigen peptides incubated for $40 \mathrm{~min}$. at $37^{\circ} \mathrm{C}$. Incorporation of TRPC antibodies into mesenteric arteries by pinocytosis was facilitated by changing the bathing solution to a hypotonic solution $(0.45 \% \mathrm{NaCl})$ for $20 \mathrm{sec}$. according to recent literature [17].

\section{Immunohistochemistry}

Fresh mesenteric arterioles were fixed with $10 \%$ formalin for $10 \mathrm{~min}$. at room temperature, washed with phosphate-buffered saline (PBS) and then permeabilized with PBS containing $0.5 \%$ Triton X-100 for 20 min. at room temperature. Mesenteric arterioles were incubated with PBS containing with $5 \%$ bovine serum and $0.1 \%$ Triton $X-100$ for $1 \mathrm{hr}$ at room temperature. Then arterioles were incubated with rabbit anti-TRPC1, anti-TRPC3 and anti-TRPC5 antibodies (1:200; Alomone Labs) over night at $4^{\circ} \mathrm{C}$. Mesenteric arterioles were then washed and incubated with secondary antibodies conjugated with a fluorescent probe (FITC-conjugated goat antirabbit antibodies; Santa Cruz Biotechnology, Delaware Avenue, CA, USA; 1:200) for $30 \mathrm{~min}$. at room temperature. In control experiments, the primary TRPC3 antibody was pre-incubated with antigenic peptide (1:25) for $12 \mathrm{hrs}$ at $4^{\circ} \mathrm{C}$. After removing the unbound secondary antibodies by washing the preparations with PBS, imaging was performed with the fluorescence microscope (Nikon, TE2000, Tokyo, Japan). 


\section{Measurement of intracellular calcium in fresh mesenteric arterioles}

PBS, with the following composition (in mmol/l) $137 \mathrm{NaCl}, 4.1 \mathrm{KCl}, 0.66$ $\mathrm{KH}_{2} \mathrm{PO}_{4}, 3.4 \mathrm{Na}_{2} \mathrm{HPO}_{4}, 2.5 \mathrm{NaHCO}_{3}, 1.0 \mathrm{MgCl}_{2}$ and 5 glucose, was adjusted daily to $\mathrm{pH} 7.4$ at room temperature. Arterioles were loaded with the fluorescent dye fura2-acetoxymethylester (final concentration, $2 \mu \mathrm{mol} / \mathrm{l}$ ) and $0.2 \%$ pluronic $\mathrm{F}-127$ for $45 \mathrm{~min}$. at $37^{\circ} \mathrm{C}$ in the dark. The arterioles were washed twice with PBS and $1.1 \mathrm{mmol} / \mathrm{l}$ calcium was added before measurements. We measured cytosolic calcium in fresh mesenteric arterioles as described by Fellner and Arendshorst [18]. Mesenteric arterioles were identified by morphology and centred in a glass of the optical field that was free of adventitia. Changes of cytosolic calcium after administration of norepinephrine were measured in the absence and presence of inhibitors or anti-TRPC antibodies.

\section{Immunoblotting}

Immunoblotting was performed as previously described by our group $[11,12]$. Mesenteric arterioles were rapidly excised and frozen in liquid nitrogen, then were crushed in liquid nitrogen and resuspended in ice-cold lysis buffer $(20 \mathrm{mmol} / \mathrm{l}$ Tris-HCl, $\mathrm{pH} 7.4,50 \mathrm{mmol} / \mathrm{l} \mathrm{NaCl}, 50 \mathrm{mmol} / \mathrm{l} \mathrm{NaF}$, $5 \mathrm{mmol} / \mathrm{l}$ ethylenediaminetetraacetic acid and $20 \mathrm{mmol} / / \mathrm{Na}_{4} \mathrm{P}_{2} \mathrm{O}_{7}$ ). Supernatants were collected, and protein concentrations were estimated. Lysates of VSMC) and supernatants from mesenteric arterioles were subjected to SDS-PAGE gel electrophoresis (10\%). Separated proteins were then electrotransferred onto nitrocellulose. Nitrocellulose membranes were blocked in $5 \%$ non-fat dry milk in Tris-buffered saline containing $0.1 \%$ Tween-20 for $60 \mathrm{~min}$. at room temperature. The membranes were incubated with primary rabbit antibodies against TRPC1, TRPC3, TRPC4, TRPC5 and TRPC6 (1:200; Alomone Labs), followed by incubation with goat anti-rabbit horseradish peroxidase-conjugated secondary antibody. The specific bands were quantified using an image analyser (Biorad Laboratories, Philadelphia, PA, USA) .

\section{Statistics}

Data are given as mean \pm S.E.M. Statistical analyses of the data were performed with the two-tailed Student's t-test for unpaired comparisons. Analysis of data Anova was used, because vasomotion was investigated in several unrelated vessels when comparing more than two groups. Values were considered to be significant when two-sided $P$ was less than 0.05 .

\section{Results}

\section{Increased norepinephrine-induced vasomotion in mesenteric arterioles from SHR}

After administration of $10 \mu \mathrm{mol} / /$ norepinephrine a rapid vasoconstriction was followed by synchronized oscillations of vascular tone, i.e. vasomotion, in mesenteric arterioles from WKY (Fig. 1A) and SHR (Fig. 1B). Norepinephrine-induced vasomotion was quantified as percentages of oscillations of vascular tone in relation to the maximal contraction to $\mathrm{KCl}$. (Fig. 1C). The norepinephrine-induced vasomotion was significantly higher in mesenteric arterioles from SHR compared to WKY $(65.5 \pm 7 \%$ versus $2.2 \pm$ $0.5 \%$; each $n=6, P<0.01$ ). The norepinephrine-induced vasoconstriction was also significantly increased in mesenteric arterioles from SHR compared to WKY (140 $\pm 11 \%$ versus $100 \pm 3 \%$; each $n=6, P<0.05$, Fig. 1D). Recordings for more than $1 \mathrm{hr}$ confirmed increased norepinephrine-induced vasomotion in SHR compared to WKY (Fig. 1E and F).

\section{Increased expression of TRPC1, TRPC3 and TRPC5 channels in mesenteric arterioles from SHR}

Using immunoblotting we demonstrated the expression of TRPC1, TRPC3, TRPC4, TRPC5 and TRPC6 channels in mesenteric arterioles (Fig. 2A). Immunoblottings showed that the expressions of TRPC1, TRPC3 and TRPC5 channels were significantly higher in mesenteric arteries from SHR compared to WKY (TRPC1 expression for WKY $1.00 \pm 0.13$ versus $1.60 \pm 0.10$ for SHR; $P<0.01$; TRPC3 expression for WKY $1.00 \pm 0.18$ versus $1.70 \pm 0.26$ for SHR; $P<0.05$; TRPC5 expression for WKY $1.00 \pm 0.13$ versus $1.41 \pm 0.10$ for SHR; $P<0.05$; each $n=6$; Fig. $2 \mathrm{~B})$. On the other hand, the expressions of TRPC4 and TRPC6 channels were not significantly different between the strains (TRPC4 expression for WKY $1.00 \pm 0.09$ versus $1.16 \pm 0.08$ for SHR; TRPC6 expression for WKY $1.00 \pm 0.18$ versus $1.09 \pm 0.06$ for SHR; each $n=6$; $P>0.05)$. After pre-incubation of TRPC3 antibodies with their respective antigen no bands could be observed any longer. Immunohistochemistry confirmed the expression of TRPC1, TRPC3 and TRPC5 were significantly increased in mesenteric arterioles from SHR compared to WKY (Fig. 2C SHR and Fig. 2D WKY).

\section{Inhibition of TRPC significantly attenuates norepinephrine-induced vasomotion in SHR}

After calcium-depletion of sarcoendoplasmic reticulum calcium stores by $1 \mu \mathrm{mol} / \mathrm{l}$ thapsigargin in the absence of extracellular calcium, the administration of extracellular calcium and norepinephrine repeatedly induced vasomotion in mesenteric arterioles from SHR. In the absence of calcium the thapsigarin-induced calcium increase was $76 \pm 13 \mathrm{nmol} / \mathrm{l}$. After return of cytosolic calcium to baseline values the subsequent administration of norepinephrine did not significantly change cytosolic calcium from $65 \pm 12 \mathrm{nmol} / \mathrm{l}$ to $70 \pm 12 \mathrm{nmol} / \mathrm{l} ; n=10 ; P>0.05)$. These data indicate that intracellular stores are empty after administration of thapsigargin. As shown in Fig. 3, compared to control conditions (57.0 $\pm 6 \%$, $n=6$, Fig. $3 \mathrm{~A}$ ), the norepinephrine-induced vasomotion was significantly reduced in the presence of several chemically 
A

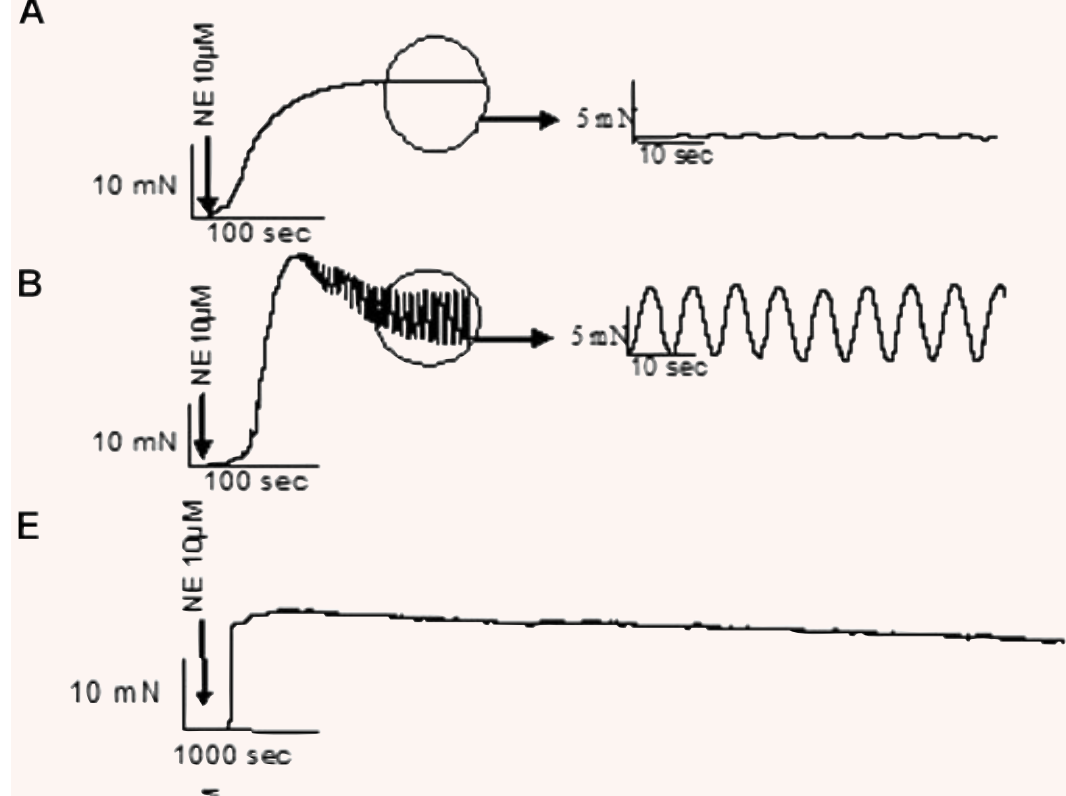

C

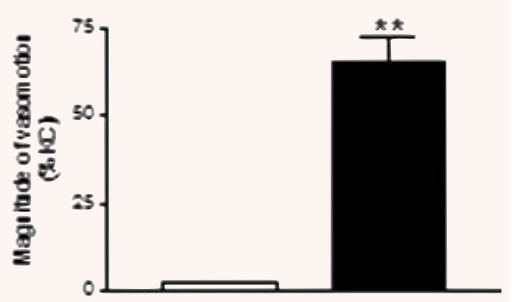

D

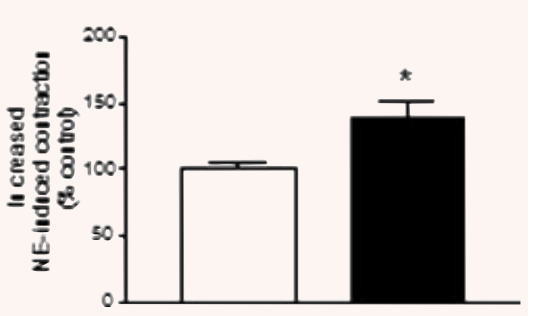

$\mathrm{F}$

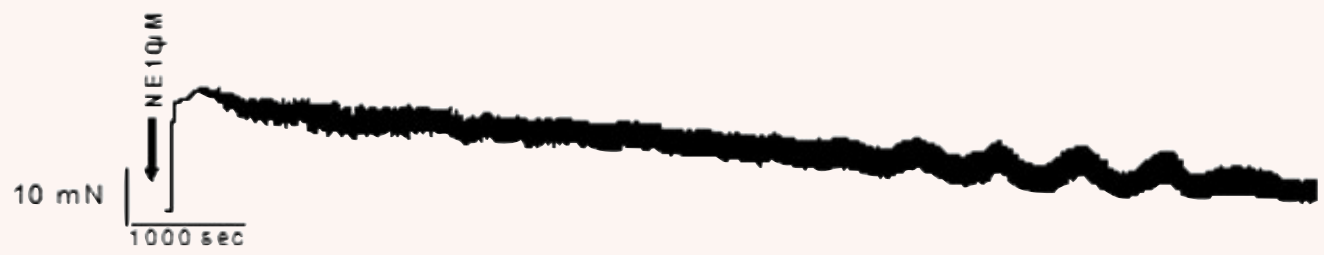

Fig. 1 Comparison of vasomotion induced by norepinephrine $(10 \mu \mathrm{mol} / \mathrm{l})$ in mesenteric arterioles from WKY (A) and SHR (B). (C) Summary data for norepinephrine-induced vasomotion in mesenteric arterioles from SHR (filled bars) compared with WKY (open bars). (D) Summary data for norepinephrine-induced vasoconstriction in mesenteric arterioles from SHR (filled bars) compared with WKY (open bars). Data are given as mean \pm S.E.M. ${ }^{\star \star} P<0.01$ or ${ }^{\star} P<0.05$ by two-tailed Student's t-test. Representative recordings lasting for more than $1 \mathrm{hr}$ showing norepinephrine-induced vasomotion in mesenteric arterioles from WKY $(\mathbf{E})$ or SHR (F).

unrelated TRPC blockers, i.e. in the presence of gadolinium reduced to $48.5 \pm 6 \%$ (Fig. $3 \mathrm{~B}$ ), in the presence of 2-APB reduced to $30.3 \pm 3 \%$ (Fig. $3 \mathrm{C}$ ) and in the presence of SKF96365 reduced to $23.5 \pm 9 \%$ (Fig. 3D) (each $n=6$; ${ }^{\star} P<0.05$ or ${ }^{\star}{ }^{\star} P<0.01$ by ANOVA, Fig. 3I). On the other hand, vasomotion may be evoked and affected by transplasmamembrance calcium influx through several different types of calcium channels. To distinguish the effects of different channel types in the present study we used blocker for TRP channels as well as verapamil. We evaluated effects of verapamil on norepinephrine-induced vasomotion. The experiments indicated that verapamil also reduces norepinephrine-induced vasomotion. However, the inhibitory effect of verapamil plus gadolinium, verapamil plus 2-APB, or verapamil plus SKF96365 was more pronounced. As shown in Fig. $3(\mathrm{E})-(\mathrm{H})$, in the presence of verapamil (final concentration $10^{-7} \mathrm{~mol} / \mathrm{l}$ ), the norepinephrine-induced vasomotion was significantly reduced to $30.0 \pm 5 \%$ of the maximal contraction to $\mathrm{KCl}$ (Fig. 3E) compared to control conditions. It should be noted that in the presence of verapamil plus $10^{-4} \mathrm{~mol} / / \mathrm{Gd}^{3+}$ (Fig. 3F), verapamil plus $10^{-5}$ $\mathrm{mol} / \mathrm{l}$ 2-APB (Fig. 3G) or verapamil plus $10^{-5} \mathrm{~mol} / \mathrm{l}$ SKF96365 (Fig. $3 \mathrm{H}$ ), the norepinephrine-induced vasomotion was significantly reduced to $18.2 \pm 5 \%, 13.7 \pm 4 \%$, or $2.9 \pm 0.5 \%$ of the maximal contraction to $\mathrm{KCl}$, respectively (each $n=6$, ${ }^{\star} P<0.05$ or ${ }^{* *} P<0.01$ compared to control conditions).

As previously indicated by Andresen and Yang [19] and Hajduczok [20] we occasionally noted that $\mathrm{Gd}^{3+}$ at a concentration of $1 \mathrm{mmol} / \mathrm{l}$ turned the buffer solution slightly cloudy probably due to gadolinium precipitation. Additional experiments comparing the gadolinium effects using cells suspended in HEPES-buffered solution in the absence and presence of 0.78 $\mathrm{mmol} / \mathrm{l}$ phosphate were performed. The effect of gadolinium on vasomotion was not significantly different in the absence or presence of $0.78 \mathrm{mmol} / /$ phosphate $(48.5 \pm 6 \%$ versus $49.3 \pm 7 \%$.; $n=6 ; P>0.05)$. 
A

B
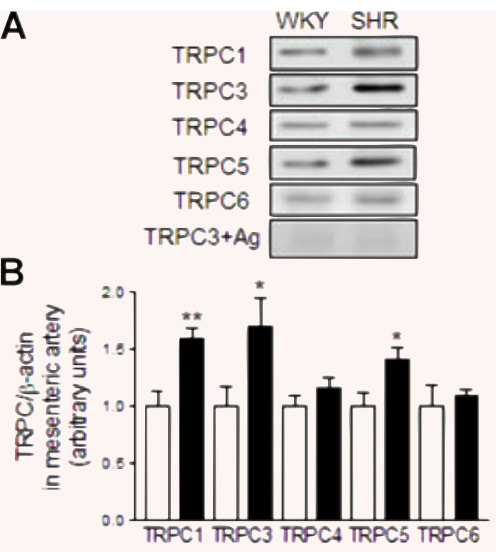

C

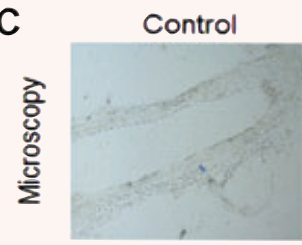

TRPC

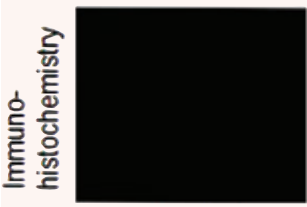

Control

D
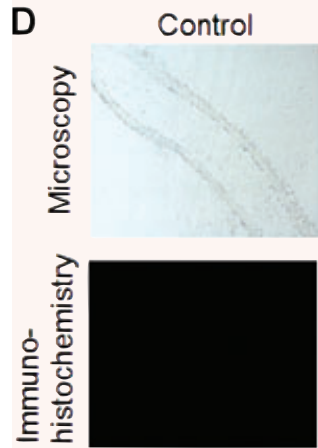

Control
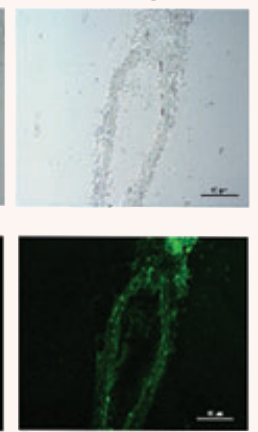

TRPC1
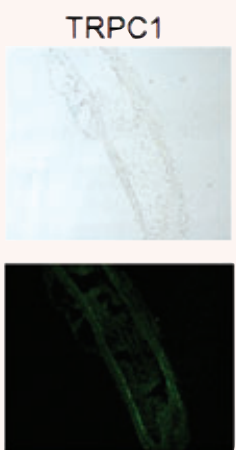

TRPC1
TRPC3
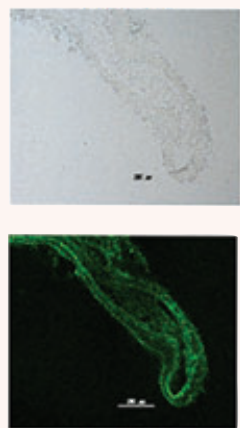

TRPC3
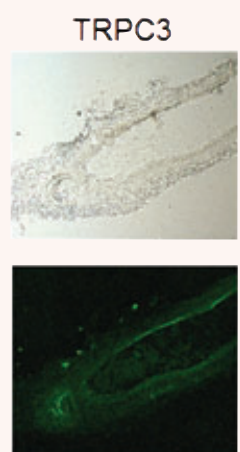

TRPC3
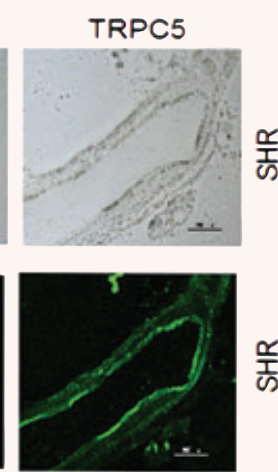

TRPC5 TRPC5
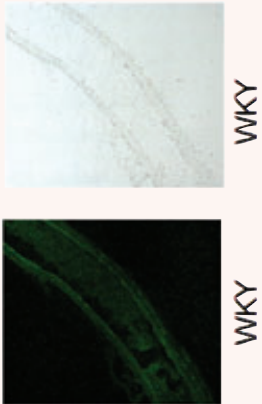

TRPC5

Fig. 2 Expression of TRPC1, TRPC3 and TRPC5 channels in mesenteric arterioles from SHR. Representative immunoblottings (A) and summary data (B) of TRPC1, TRPC3, TRPC4, TRPC5,TRPC6 channels and TRPC3 antibody with its respective antigenic peptide the protein expression in mesenteric arterioles from WKY (open bars) and SHR (filled bars). Data are mean \pm S.E.M. of $n=4$ independent experiments. ${ }^{*} P<0.05$ or ${ }^{* *} P<0.01$ by two-tailed Student's t-test. (C) Light microscopy of mesenteric arterioles from SHR (upper panels) and immunohistochemistry (lower panels) of TRPC1, TRPC3 and TRPC5 protein expression in mesenteric arterioles from SHR. (D) Light microscopy of mesenteric arterioles from WKY (upper panels) and immunohistochemistry (lower panels) of TRPC1, TRPC3 and TRPC5 protein expression in mesenteric arterioles from WKY. Immunohistochemistry using specific antibodies labelled with green shows TRPC1, TRPC3 and TRPC5 protein expression in mesenteric arterioles. Control indicates immunohistochemistry after pre-incubation of the primary TRPC3 antibody with antigenic peptide for $12 \mathrm{hrs}$ at $4^{\circ} \mathrm{C}$. Bar denotes $200 \mu \mathrm{m}$.

\section{Inhibition of norepinephrine-induced vasomotion in mesenteric arterioles from SHR by specific TRPC antibodies}

We also examined the effects of specific anti-TRPC antibodies on norepinephrine-induced vasomotion in mesenteric arterioles from SHR. Under control conditions, i.e. in the absence of TRPC antibodies, the norepinephrine-induced vasomotion was $57.0 \pm 6 \%$ of the maximal contraction to $\mathrm{KCl}$ (Fig. 4A). Compared to control conditions, the norepinephrine-induced vasomotion was signifi- cantly reduced in the presence of anti-TRPC1 antibodies to $32.0 \pm$ $7 \%(P<0.05 ; n=6$, Fig. 4B $)$ and in the presence of anti-TRPC3 antibodies to $30.0 \pm 7 \%(P<0.05 ; n=6$, Fig. 4C). On the other hand, to support the specific effects of anti-TRPC antibodies on norepinephrine-induced vasomotion additional control experiments were performed with the respective antigenic peptides. Preincubation with respective antigenic peptide significantly abolished the inhibitory effect of TRPC antibodies on norepinephrineinduced vasomotion. In the presence of TRPC3 antigenic peptide and anti-TRPC3 antibody the norepinephrine-induced vasomotion was not significantly different compared to control conditions 


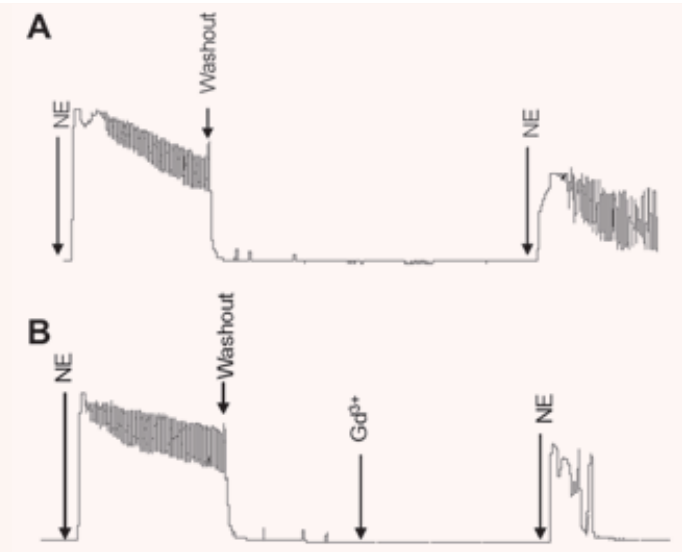

E

宸

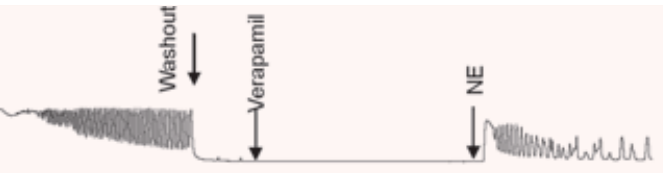

$\mathbf{F}$

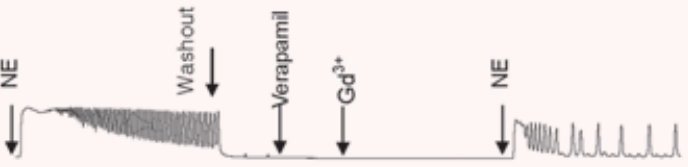

G
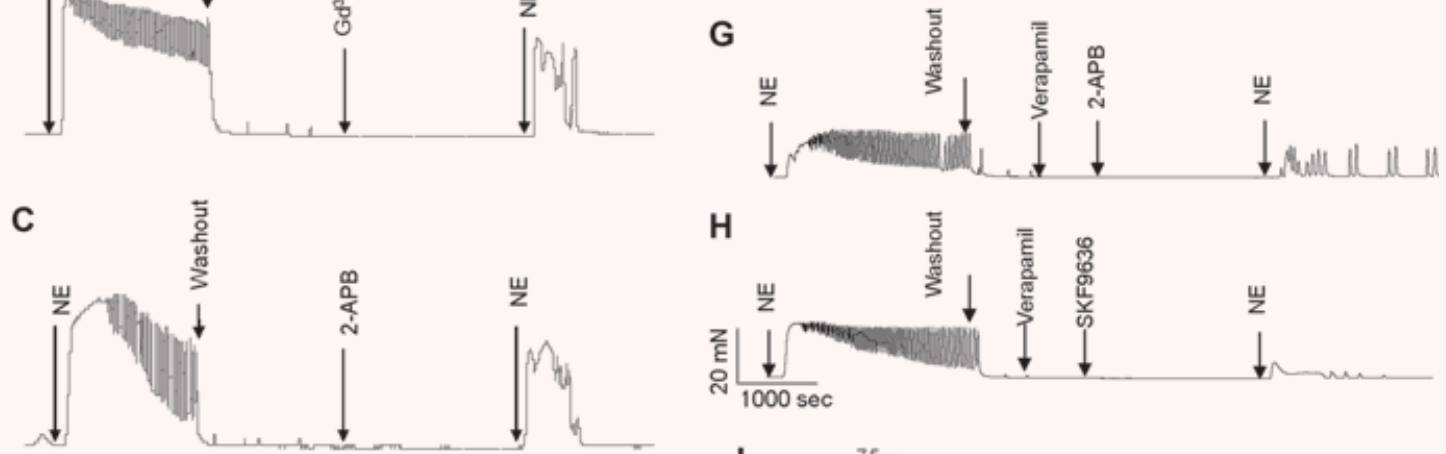

H
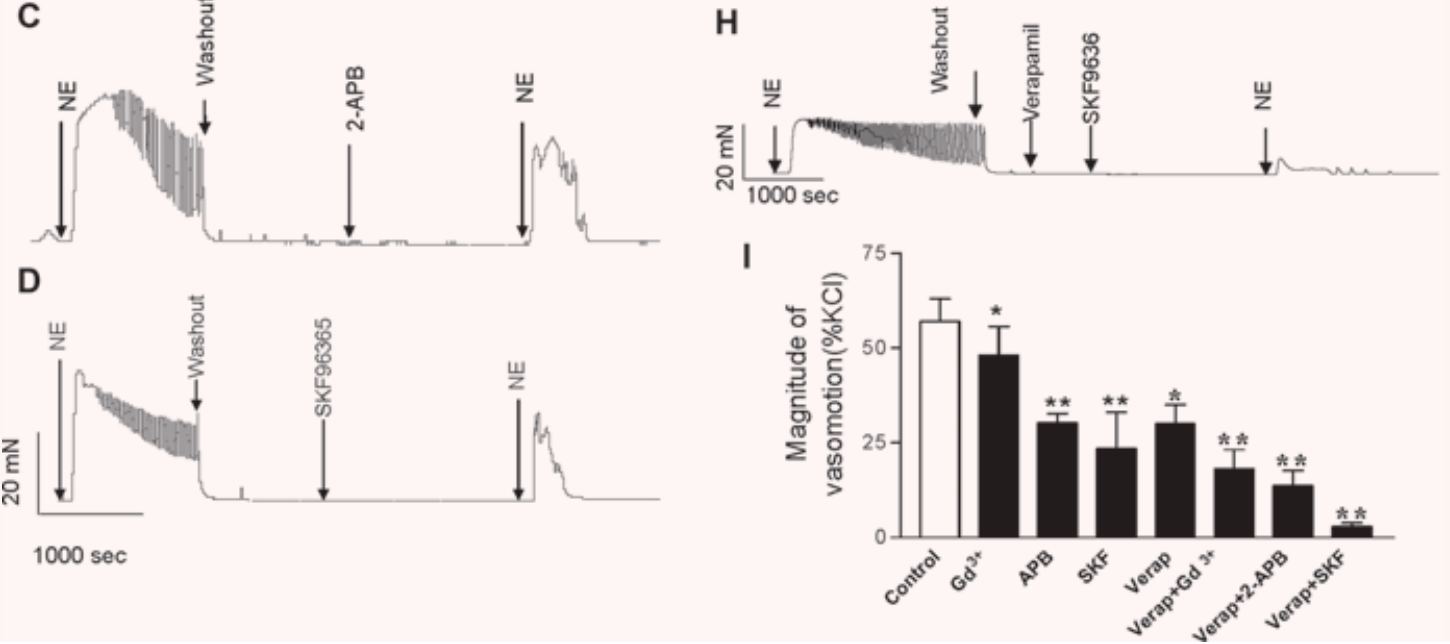

Fig. 3 Inhibition of TRPC significantly attenuates norepinephrine-induced vasomotion in SHR. Vasomotion was induced by norepinephrine after depletion of sarcoendoplasmic reticulum calcium stores by $1 \mu \mathrm{mol} / \mathrm{l}$ thapsigargin (TG) in mesenteric arterioles from SHR under control conditions (A), in the presence of gadolinium $\left(\mathrm{Gd}^{3+}\right)(\mathbf{B}), 2-\mathrm{APB}(\mathbf{C})$ or SKF96365 (D), in the presence of verapamil (E), or in the presence of verapamil plus Gd ${ }^{3+}(\mathbf{F})$, verapamil plus 2-APB (G) or verapamil plus SKF96365 (H). (I) Summary data are shown as mean \pm S.E.M. of $n=6$ independent experiments. ${ }^{*} P<0.05$ or ${ }^{* *} P<0.01$ by ANOVA.

(43.0 $\pm 15 \% ; P=$ n.s.; $n=6$, Fig. 4D). In the presence of antiTRPC5 antibodies the norepinephrine-induced vasomotion was not significantly affected $(45.0 \pm 11 \% ; P=$ n.s. Fig. $4 \mathrm{E})$. In the presence of anti-TRPC1 plus anti-TRPC3 antibodies the norepinephrine-induced vasomotion was significantly reduced to $29.0 \pm$ $8 \%(P<0.05 ; n=6$, Fig. 4F). Furthermore, pre-incubation of mesenteric arterioles with anti- $\beta$-actin antibodies did not significantly affect norepinephrine-induced vasomotion when compared to control conditions $(59.8 \pm 15 \%, P=$ n.s.; $n=6$, Fig. 4G). Incorporation of TRPC antibodies into mesenteric arteries by pinocytosis was facilitated by changing the bathing solution to a hypotonic solution according to recent literature [17]. The magnitude of vasomotion of the maximal contraction to $\mathrm{KCl}$ was
$57.6 \pm 5 \%$ under control conditions with isotonic $\mathrm{NaCl}$, it was $54.0 \pm 7 \%$ under control conditions after short-term exposure to hypotonic $0.45 \% \mathrm{NaCl}$ without antibody, and it was $23.4 \pm 4 \%$ after short-term exposure to hypotonic $0.45 \% \mathrm{NaCl}$ plus TRPC3 antibody $(P<0.01$.; $n=6$, Fig. $4 \mathrm{H}-\mathrm{J})$.

Control experiments were performed to underline the significance of using TRPC antibodies for inhibition of norepinephrineinduced vasomotion. First, destruction of the antibody by heating abolished its effects on vasomotion. After heating of the antiTRPC3 antibody to $95^{\circ} \mathrm{C}$ for $5 \mathrm{~min}$. no effect on norepinephrineinduced vasomotion could be observed $(58.0 \pm 7 \%$ versus $56.3 \pm$ $5 \%$, each $n=6 ; P>0.05$ ). Second, we compared TRPC antibodies supplied from different sources. Additional experiments using 
A

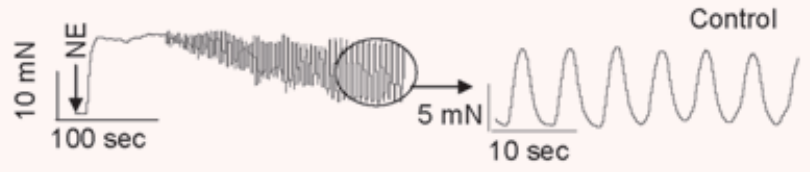

B

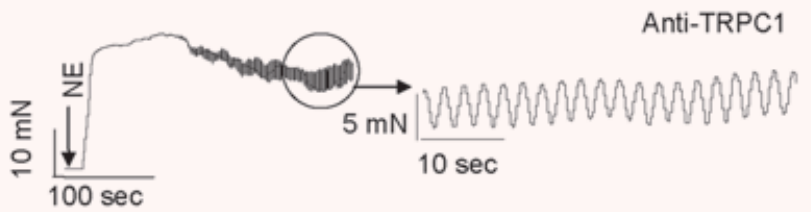

C

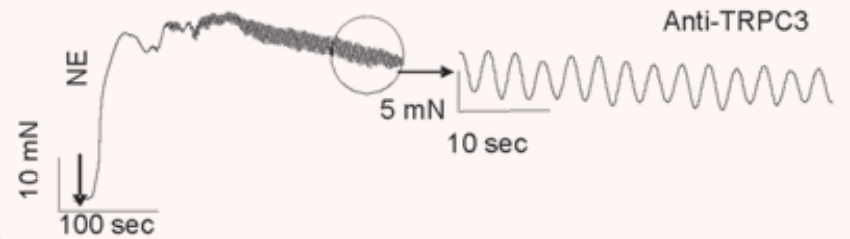

D

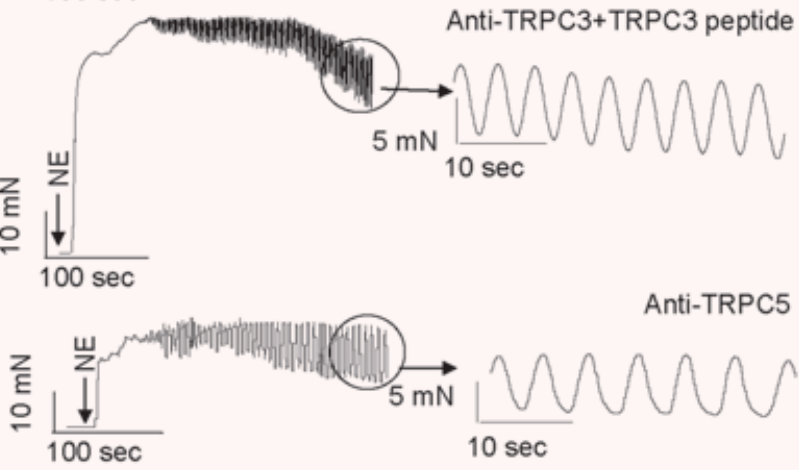

F

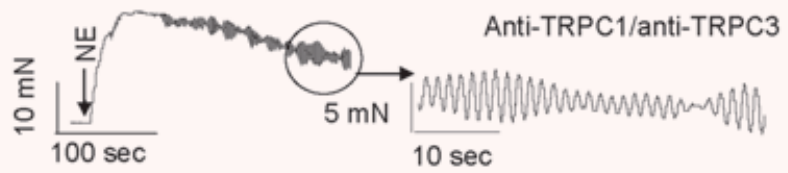

G

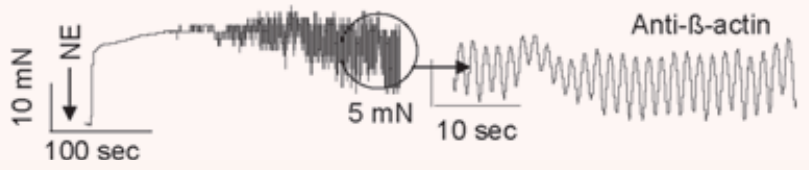

H

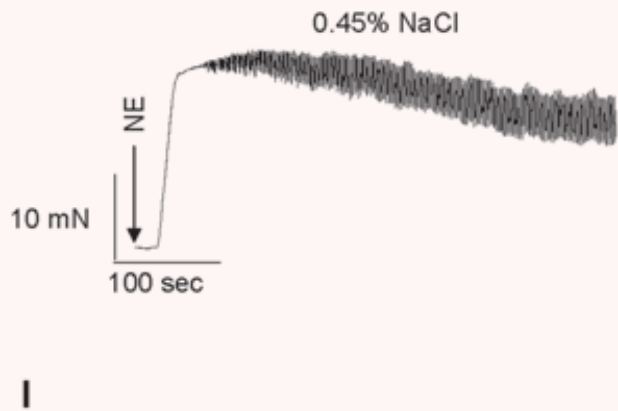

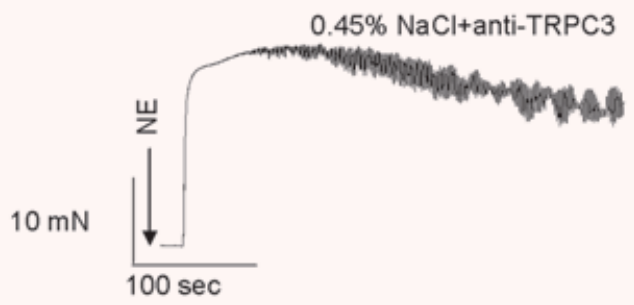

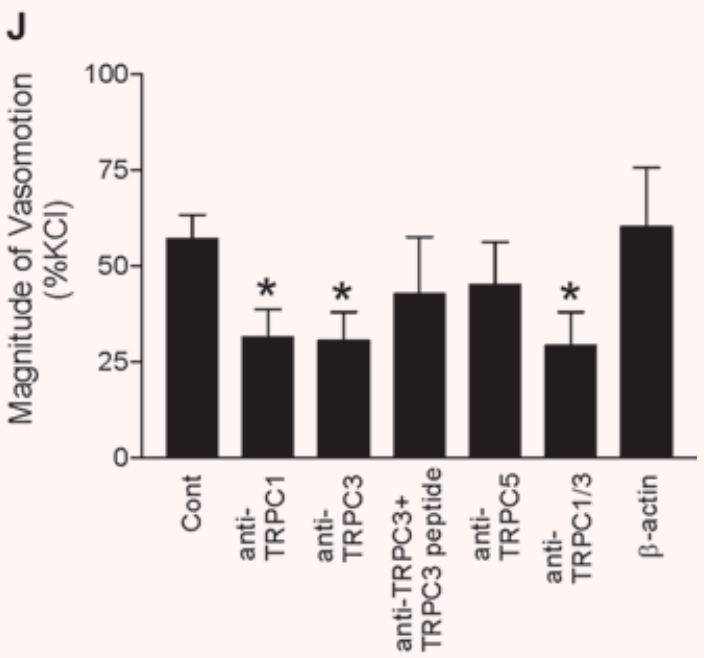

Fig. 4 Inhibition of norepinephrine-induced vasomotion in mesenteric arterioles from SHR by specific anti-TRPC antibodies Representative tracings of norepinephrine-induced vasomotion in mesenteric arterioles from SHR under control conditions (A), in the presence of anti-TRPC1 antibodies (B), antiTRPC3 antibodies (C), anti-TRPC3 antibodies with antigenic peptide (D), anti-TRPC5 antibodies (E), anti-TRPC1 plus anti-TRPC3 antibodies (F) or in the presence of anti- $\beta$-actin antibodies $(\mathbf{G})$. Representative tracings of norepinephrine-induced vasomotion in mesenteric arterioles from SHR under control conditions after short-term exposure to hypotonic $0.45 \% \mathrm{NaCl}$ without antibody $(\mathbf{H})$, or after short-term exposure to hypotonic $0.45 \% \mathrm{NaCl}$ plus TRPC3 antibody (I). Summary data (J) show that inhibition of TRPC channels by specific anti-TRPC antibodies significantly attenuates norepinephrineinduced vasomotion in SHR. Data are mean \pm S.E.M. of $n=6$ independent experiments. ${ }^{*} P<0.05$ by ANOvA. 
anti-TRPC3 antibodies from a different supplier also showed the reduction of norepinephrine-induced vasomotion in mesenteric arteries to $30.4 \pm 9 \%$ of the maximal contraction to $\mathrm{KCl}$ ( $n=6 ; P<0.05$ compared to control). Third, we used random immunoglobulins not related to TRPC channels. Administration of random immunoglobulins did not affect norepinephrine-induced vasomotion (53.0 $\pm 7 \% ; n=4, P>0.05$ compared to control), thus excluding non-specific effects of immunoglobulins. Furthermore, exposure of a high albumin concentration did not affect the norepinephrine-induced vasomotion in mesenteric arteries from SHR (55.4 $\pm 7 \% ; n=4 ; P>0.05$ compared to control). These data underline that specific interactions with TRPC channels can modify norepinephrine-induced vasomotion.

\section{Inhibition of norepinephrine-induced calcium increase in mesenteric arterioles from SHR by specific TRPC antibodies}

We further evaluated the effects of specific anti-TRPC antibodies on norepinephrine-induced calcium increase in mesenteric arterioles. As shown in Fig. 5, under control conditions, the norepinephrine-induced calcium increase in mesenteric arterioles was $299 \pm 20 \mathrm{nmol} / \mathrm{l}(n=8)$. In the presence of anti-TRPC1 antibodies, the norepinephrine-induced calcium increase was significantly reduced to $150 \pm 31 \mathrm{nmol} / \mathrm{l}(P<0.01 ; n=8$, Fig. $5 \mathrm{~A})$. In the presence of anti-TRPC3 antibodies, the norepinephrine-induced calcium increase was significantly reduced to $188 \pm 37 \mathrm{nmol} / \mathrm{l}$ $(P<0.05 ; n=8$, Fig. 5B). In the presence of anti-TRPC5 antibodies, the norepinephrine-induced calcium increase was significantly reduced to $185 \pm 48 \mathrm{nmol} / \mathrm{l}(P<0.05 ; n=8$, Fig. $5 \mathrm{C})$. In the presence of anti-TRPC1 plus anti-TRPC3 and anti-TRPC5 antibodies the norepinephrine-induced calcium increase was significantly reduced to $100 \pm 18 \mathrm{nmol} / \mathrm{l}(P<0.01 ; n=8$, Fig. 5D). However, in the presence of anti-TRPC3 antibodies plus TRPC3 antigenic peptide, the norepinephrine-induced calcium increase was not significantly different compared to control conditions (298 \pm $14 \mathrm{nmol} / \mathrm{l}, P>0.05 ; n=8$, Fig. 5E). Furthermore, anti- $\beta$-actin antibodies did not significantly affect norepinephrine-induced calcium increase when compared to control conditions (278 \pm $13 \mathrm{nmol} / \mathrm{l}, P>0.05 ; n=8$, Fig. 5F).

\section{Effect of candesartan or telmisartan but not amlodipine on the rhythmic oscillatory vasomotion of mesenteric arterioles}

After oral administration of candesartan ( $4 \mathrm{mg} / \mathrm{kg} /$ day), telmisar$\tan (5 \mathrm{mg} / \mathrm{kg} /$ day) or amlodipine ( $10 \mathrm{mg} / \mathrm{kg} /$ day) for 16 weeks to hypertensive rats, systolic blood pressure was significantly lower compared to placebo-treated SHR (placebo, $222 \pm 7 \mathrm{mmHg}$; candesarten, $123 \pm 7 \mathrm{mmHg}$; telmisartan $115 \pm 6 \mathrm{mmHg}$; amlodipine, $122 \pm 5 \mathrm{mmHg} ; P<0.01$; each $n=6$; Fig. $6 \mathrm{~A}$ ). After treatment with candesartan or telmisartan for 16 weeks, norepi- nephrine-induced vasomotion was significantly reduced in mesenteric arterioles compared to placebo-treated SHR. Vasomotion was $66 \pm 7 \%$ in SHR under placebo control conditions, $14 \pm 3 \%$ in SHR treated with candesartan and $8 \pm 2 \%$ in SHR treated by telmisartan ( $P<0.01$; each $n=6$; Fig. 6B). It should be noted that vasomotion was not significantly affected in amlodipinetreated SHR. Representative tracings are shown in Fig. 6(C)-(F).

\section{Effect of candesartan or telmisartan but not amlodipine on TRPC expression in mesenteric arterioles}

As shown in Fig. 7 after treatment with candesartan or telmisartan for 16 weeks, TRPC1, TRPC3 and TRPC5 channel expressions were significantly reduced in mesenteric arterioles from candesartan or telmisartan-treated SHR compared to placebo-treated SHR as control group (each $P<0.05 ; n=5$ ). It should be noted that TRPC1, TRPC3 and TRPC5 channel expression were not significantly affected in amlodipine-treated SHR compared to placebotreated SHR (each $P=$ n.s.; $n=5$ ).

\section{Discussion}

The major finding of the present study was that increased oscillations of arterial vascular tone due to synchronized changes of intracellular calcium concentrations are linked to increased TRPC expression in hypertension. We gave several experimental evidence: First, significantly increased expression of TRPC1, TRPC3 and TRPC5 channels in mesenteric arterioles from SHR compared to WKY; second, increased vasomotion in mesenteric arterioles from SHR compared to WKY; third, reduced vasomotion in the presence of TRPC blockers and anti-TRPC antibodies and fourth, reduction of TRPC expression and vasomotion in SHR after longterm administration of candesartan or telmisartan, but not amlodipine.

Vasomotion which occurs in small resistance vessels of the microcirculation, as well as in larger arteries, is expected to increase flow to assist in tissue perfusion especially during periods of altered metabolism or perfusion pressure [1]. On the other hand, increased vasomotion may be responsible for development and maintenance of pathophysiological states including hypertension [2]. The present study confirmed previous reports showing increased vasomotion in hypertension [7-9]. Earlier genetic studies based on crossbreeding showed a close association between hypertension and increased oscillations of arterial vascular tone $[21,22]$. That close association in genetic studies suggests that the underlying molecular mechanisms causing hypertension and vasomotion may be linked to each other.

Which are the underlying mechanisms of norepinephrineinduced vasomotion in mesenteric arterioles? The application of 
A

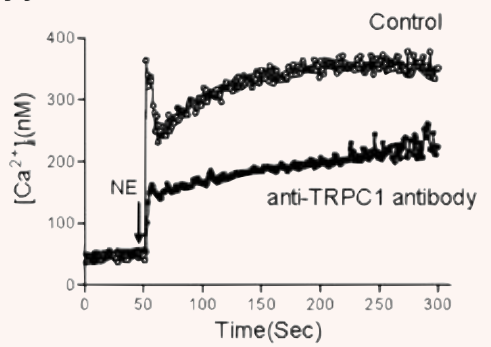

C

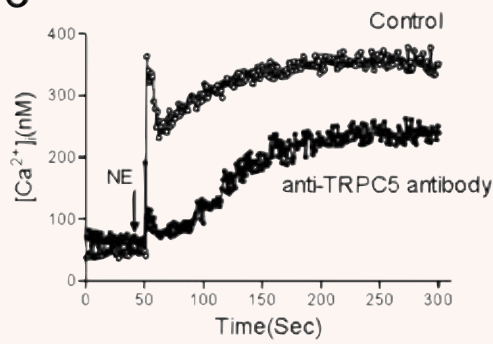

E

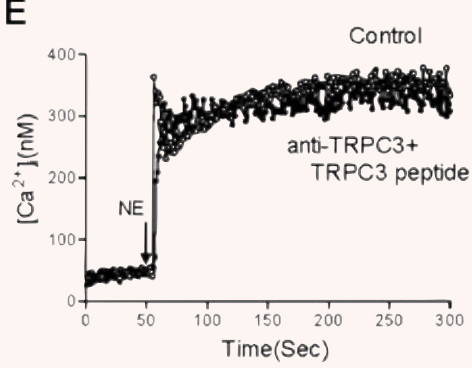

B

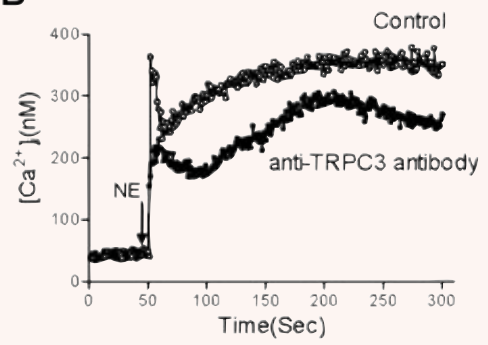

D

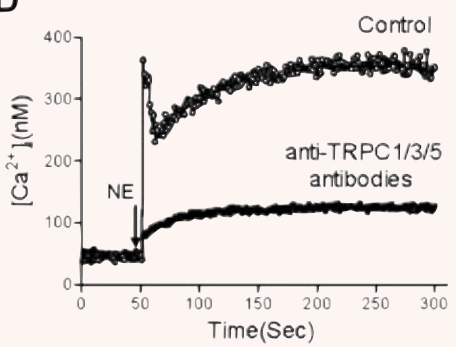

F

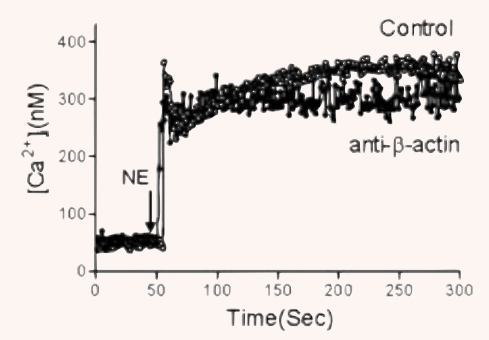

Fig. 5 Inhibition of norepinephrine-induced calcium increase in mesenteric arterioles from SHR by specific anti-TRPC antibodies. Representative tracings of norepinephrineinduced calcium increase in mesenteric arterioles from SHR under control conditions and in the presence of anti-TRPC1 antibodies (A), anti-TRPC3 antibodies (B), antiTRPC5 antibodies (C), anti-TRPC1 plus anti-TRPC3 plus anti-TRPC5 antibodies (D), anti-TRPC3 antibodies plus TRPC3 antigenic peptide (E) or anti- $\beta$-actin antibodies (F). Summary data $(\mathbf{G})$ shows the effects of specific anti-TRPC antibodies on norepinephrine-induced calcium influx in mesenteric arterioles from SHR. Data are mean \pm S.E.M. of $n=8$ independent experiments. ${ }^{*} P<0.05,{ }^{* *} P<0.01$ or $\# P>0.05$ compared to control conditions by ANOVA.
G

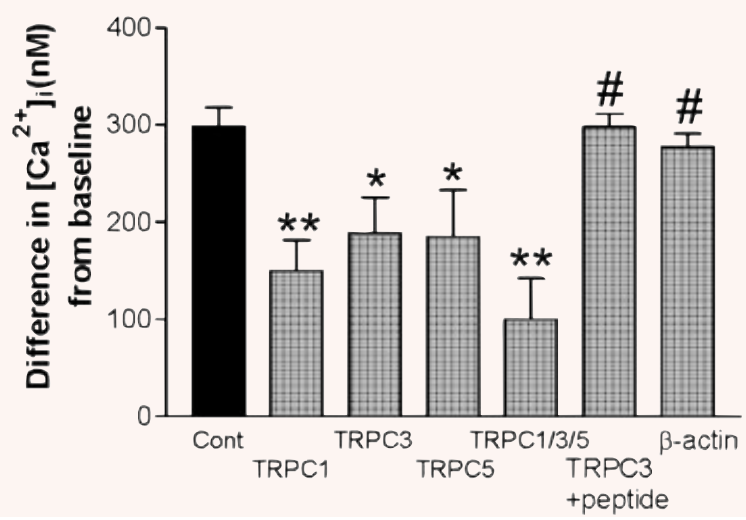

norepinephrine activates G-protein coupled receptors, and subsequent release of $G$ proteins stimulates phospholipase $C \beta 1$ to produce diacylglycerol and inositoltrisphosphate. Diacylglycerol directly activates so-called second-messenger-operated TRPC channels [22-24]. TRPC1, TRPC3 and TRPC5 channels have been reported to form second-messenger-operated calcium entry channels [22-24]. Furthermore, TRPC1, TRPC3 and TRPC5 channels show also characteristics of store-operated TRP channels [25-28].
Subsequent calcium influx or oscillatory release of calcium from intracellular stores then activates calcium-dependent chloride channels which intermittently depolarize the membrane potential $[1,2]$. Depolarization and calcium influx through voltage-dependent calcium channels has been shown to be an important step in coordinating the individual oscillators for example in rat mesenteric arteries [29]. Hence, the increased expression of TRPC channels in rat mesenteric arteries may be the initial trigger for increased 
A

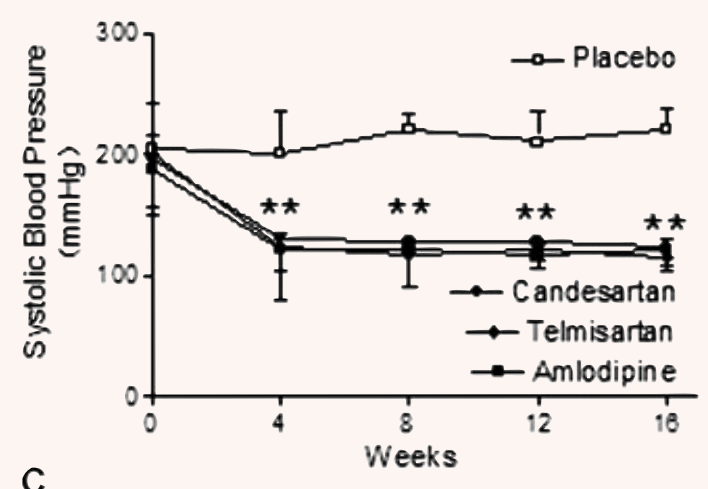

C

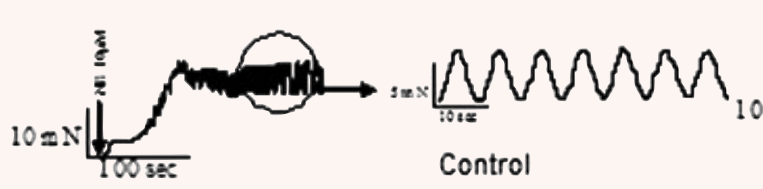

B

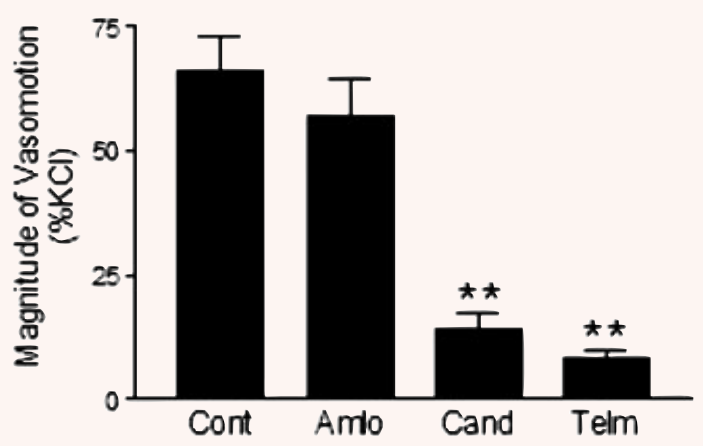

D

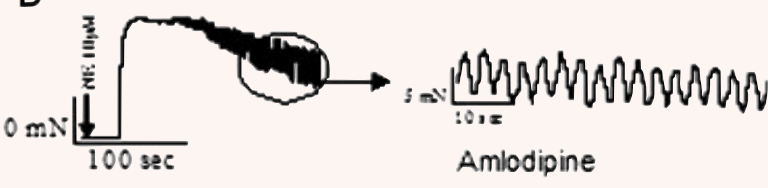

E

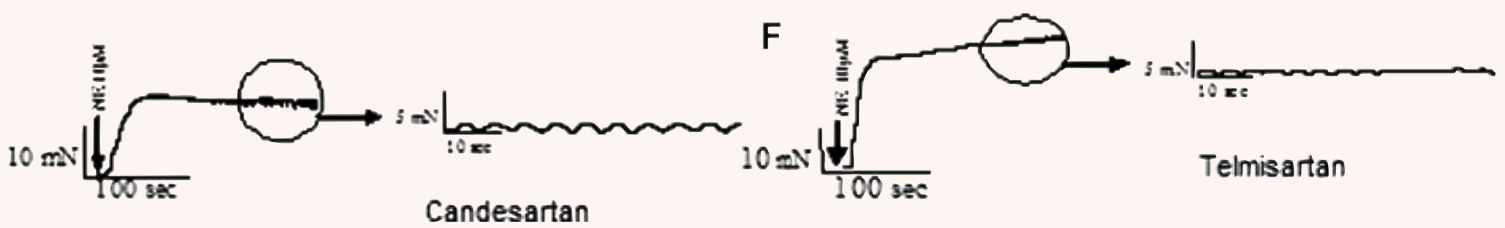

Fig. 6 Effect of candesartan or telmisartan but not amlodipine on norepinephrine-induced vasomotion in mesenteric arterioles. Long-term administration of angiotensin AT1 receptor antagonist telmisartan or candesartan or calcium channel blocker amlodipine reduces blood pressure in vivo. The angiotensin AT1 receptor antagonist telmisartan ( $5 \mathrm{mg} / \mathrm{kg}$ per day) or candisartan (4 mg/kg per day), calcium channel blocker amlodipine (10 mg/kg per day) or placebo were administered to SHR by gavage for 16 weeks. (A) Summary data for systolic blood pressure from SHR. (B) Summary data for the rhythmic oscillatory vasomotion in mesenteric arterioles from SHR after treatment with candesartan, telmisartan, or amlodipine for 16 weeks. Representative tracings of norepinephrine-induced vasomotion in mesenteric arterioles from SHR under control conditions (C), administration of amlodipine (D), candesartan $(\mathbf{E})$ or telmisartan $(\mathbf{F}) .{ }^{* *} P<0.01$ compared with placebo by ANova.

oscillations of arterial vascular tone in hypertensive vasculature. It cannot be ruled out that part of the inhibitory effects observed on norepinephrine-induced vasomotion by several inhibitors or TRPC antibodies may be mediated by their action on endothelium [2, 3]. Although antibodies against TRPC3 or TRPC5 reduced cytosolic calcium to a similar extent, their effects on vasomotion remained different, indicating specific interaction of the antibody with their respective proteins. In the present study whole cytosolic calcium was measured in mesenteric arterioles. On the other hand, oscillations of intracellular calcium can only be observed in single-cell measurements using confocal images of cytosolic calcium over time as the changes in the mean intensity of fluorescence within some regions of interest in smooth muscle cells [5].

We used several chemically unrelated TRPC blockers, including gadolinium, 2-APB, or SKF96365, as recently described by Rose et al. [30]. Several investigators reported that 2-APB or SKF-96365 are able to reduce cation influx through TRP channels [25, 31, 32].
Higher gadolinium concentrations in the micromolar range are wellknown blockers of TRPC channels [33]. However, in the present study all three chemical distinct substances which are known to affect TRPC channels also affected vasomotion. In the presence of gadolinium, SKF-96365 or 2-APB the norepinephrine-induced vasomotion was reduced to $48 \%, 30 \%$, or $24 \%$, respectively. Furthermore, we showed that verapamil blocked norepinephrineinduced vasomotion, but the inhibitory effect of verapamil plus gadolinium, or plus 2-APB, or plus SKF96365 was more pronounced, indicating that verapamil and TRP blockers showed additive effects, whereas administration of several TRP blockers did not show additive effects. Although several investigators used TRPC channel blockers including 2-APB and SKF96365 [23, 29, 30], limitations of the specificity of inhibitory agents of TRPC channels including gadolinium, 2-APB or SKF96365 have been shown. However, all three chemical distinct substances which are known to affect TRPC channels also affected vasomotion in the present study. 


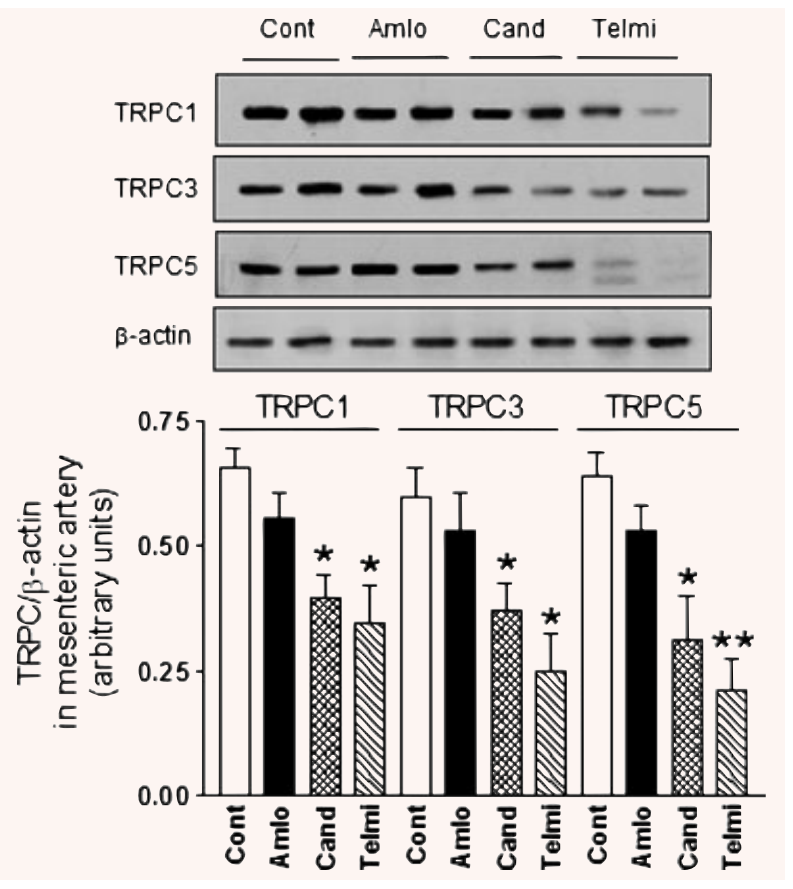

Fig. 7 Effect of candesartan or telmisartan but not amlodipine on TRPC expression in mesenteric arterioles. Long-term administration of angiotensin AT1 receptor antagonist telmisartan or candesartan, but not of calcium channel blocker amlodipine reduces TRPC1, TRPC3 and TRPC5 channel protein expression in vivo. The angiotensin AT1 receptor antagonist telmisartan ( $5 \mathrm{mg} / \mathrm{kg}$ per day) or candesartan $(4 \mathrm{mg} / \mathrm{kg}$ per day), calcium channel blocker amlodipine (10 mg/kg per day), or placebo were administered to SHR by gavage for 16 weeks. Representative immunoblottings of TRPC channel protein expressions in mesenteric arterioles from treated SHR (Fig. 7A) and summary data are shown (Fig. 7B). ${ }^{*} P<0.05 ;{ }^{*} P<0.01$ compared with placebo by ANOVA.

Furthermore we confirmed the cooperation of TRPC channels for norepinephrine-induced vasomotion using anti-TRPC antibodies. Using these commercially available anti-TRPC antibodies Peppiatt-Wildman et al. and Saleh et al. reported a high degree of selectivity for TRPC channels $[15,16]$. Furthermore, they found that anti-TRPC1 antibodies produced marked inhibition of storeoperated calcium activity in mesenteric arteries $[15,16]$. Now we showed that in the presence of anti-TRPC1 antibodies or antiTRPC3 antibodies the norepinephrine-induced vasomotion was reduced to $32 \%$ or $30 \%$, respectively. Recent study confirmed that incorporation of macromolecules including antibodies into mesenteric arteries is facilitated by changing the bathing solution to hypotonic solutions [17]. Our experiments using facilitated loading of TRPC antibodies into mesenteric arteries underscored the importance of TRPC channels for vasomotion. These experiments extended our findings that TRPC channels are associated with vasomotion.
On the other hand, control experiments in the presence of anti- $\beta$-actin antibodies showed no effect. Using random immunoglobulins which were not related to TRPC channels or using a high albumin concentration did not affect norepinephrine-induced vasomotion. Furthermore, control experiments in the presence of anti- $\beta$-actin antibodies, in the presence of random immunoglobulins or high albumin concentrations and finally pre-incubation of anti-TRPC antibodies with their respective antigenic peptides excluded the possibility of non-specific effects on vasomotion. We showed that TRPC antibodies may reduce vasomotion. However, the physicochemical properties of the antibodies may affect their inhibitory function. It may be suggested that antibodies may cross the membrane to exert the inhibitory effects, whereas the antibody-antigen complexes cannot. These results finally indicate that activation of increased TRPC expression in rat mesenteric arteries triggers increased vasomotion in hypertensive vasculature.

In the present study we used several drugs for chronic treatment of rats in order to evaluate whether lowering of blood pressure per se or the specific interaction with the angiotensin receptor was necessary to show any effect on TRPC expression and vasomotion. To answer that question we used different types of drugs. Our study showed an association between reduced TRPC expression and reduced vasomotion after long-term administration of angiotensin AT1 receptor blockers, candesartan or telmisartan, but not after administration of the calcium channel blocker, amlodipine. These findings were in accordance with earlier reports, indicating that vasomotion seemed to be less prevalent in hypertensive rats treated with angiotensin converting enzyme inhibitors [34, 35]. However, these earlier studies did not investigate TRPC channel expression. Previous results and our present findings may endorse a role for angiotensin II for the regulation of TRPC expression in vasculature and vasomotion. Furthermore, since vasomotion was not affected in the amlodipine group, even though blood pressure values were similarly reduced in the candesartan, telmisartan and amlodipine groups, changes of molecular structures including TRPC expression observed in hypertension rather than blood pressure level per se may be important for the observed coincidence of hypertension and increased vasomotion. Our results indicated that lowering blood pressure alone was not sufficient to affect TRPC expression and vasomotion. But blocking of angiotensin receptors reduced blood pressure, TRPC expression and vasomotion.

In conclusion we gave experimental evidence that the increased TRPC1, TRPC3 and TRPC5 expression in mesenteric arterioles from SHR causes increased vasomotion in hypertension.

\section{Acknowledgements}

This study was supported by grants 2006CB503905 and 2006CB503804 from 973 program (Z.Z.) and grant 81070208 from the National Natural Science Foundation of China (D.L.). 


\section{References}

1. Haddock RE, Hill CE. Rhythmicity in arterial smooth muscle. J Physiol. 2005; 566: 645-56.

2. Nilsson H, Aalkjaer C. Vasomotion: mechanisms and physiological importance. Mol Interv. 2003; 3: 79-89.

3. Seppey D, Sauser R, Koenigsberger M, et al. Does the endothelium abolish or promote arterial vasomotion in rat mesenteric arteries? Explanations for the seemingly contradictory effects. J Vasc Res. 2008; 45: 416-26.

4. Oishi H, Schuster A, Lamboley M, et al. Role of membrane potential in vasomotion of isolated pressurized rat arteries. Life Sci. 2002; 71: 2239-48.

5. Peng $H$, Matchkov V, Ivarsen $A$, et al. Hypothesis for the initiation of vasomotion. Circ Res. 2001; 88: 810-15.

6. Rahman A, Matchkov V, Nilsson H, et al. Effects of CGMP on coordination of vascular smooth muscle cells of rat mesenteric small arteries. J Vasc Res. 2005; 42: 301-11.

7. Lefer DJ, Lynch CD, Lapinski KC, et al. Enhanced vasomotion of cerebral arterioles in spontaneously hypertensive rats. Microvasc Res. 1990; 39: 129-39.

8. Osol G, Halpern W. Spontaneous vasomotion in pressurized cerebral arteries from genetically hypertensive rats. $A m \mathrm{~J}$ Physiol. 1988; 254: H28-33.

9. Zhang J, Fu S, Liu S, et al. The therapeutic effect of Ginkgo biloba extract in SHR rats and its possible mechanisms based on cerebral microvascular flow and vasomotion. Clin Hemorheol Microcirc. 2000; 23: 133-8.

10. Nilius B, Owsianik G, Voets $T$, et al. Transient receptor potential cation channels in disease. Physiol Rev. 2007; 87: 165-217.

11. Liu DY, Scholze A, Kreutz $\mathrm{R}$, et al. Monocytes from spontaneously hypertensive rats show increased store-operated and second messenger-operated calcium influx mediated by transient receptor potential canonical type 3 channels. Am J Hypertens. 2007; 20: 1111-8.

12. Liu D, Yang D, He H, et al. Increased transient receptor potential canonical type 3 channels in vasculature from hypertensive rats. Hypertension. 2009; 53; 70-6.

13. Dietrich A, Mederos Y, Schnitzler M, et al. Increased vascular smooth muscle contractility in TRPC6-/- mice. Mol Cell Biol. 2005; 25: 6980-9.
14. Huang $Y$, Chan FL, Lau CW, et al. Roles of cyclic AMP and $\mathrm{Ca}^{2+}$-activated $\mathrm{K}^{+}$ channels in endothelium-independent relaxation by urocortin in the rat coronary artery. Cardiovasc Res. 2003; 57: 824-33.

15. Zhu Z, Tepel M, Neusser M, et al. Effect of captopril on vasoconstriction and $\mathrm{Ca}^{2+}$ fluxes in aortic smooth muscle. Hypertension. 1993; 22: 806-11.

16. Peppiatt-Wildman CM, Albert AP, Saleh SN, et al. Endothelin-1 activates a $\mathrm{Ca}^{2+}$. permeable cation channel with TRPC3 and TRPC7 properties in rabbit coronary artery myocytes. J Physiol. 2007; 580: 755-64.

17. Saleh SN, Albert AP, Peppiatt-Wildman $\mathrm{CM}$ et al. Diverse properties of store-operated TRPC channels activated by protein kinase $C$ in vascular myocytes. $J$ Physiol. 2008; 586: 2463-76.

18. Mather S, Dora KA, Sandow SL, et al. Rapid endothelial cell-selective loading of connexin 40 antibody blocks endotheliumderived hyperpolarizing factor dilation in rat small mesenteric arteries. Circ Res. 2005; 97: 399-407.

19. Fellner SK, Arendshorst WJ. Store-operated $\mathrm{Ca}^{2+}$ entry is exaggerated in fresh preglomerular vascular smooth muscle cells of SHR. Kidney Int. 2002; 61: 2132-41.

20. Andresen MC, Yang M. Gadolinium and mechanotransduction of rat aortic baroreceptors. Am J Physiol. 1992; 262: 1415-21.

21. Hajduczok G, Chapleau MW, Ferlic RJ, et al. Gadolinium inhibits mechanoelectrical transduction in rabbit carotid baroreceptors. Implication of stretchactivated channels. J Clin Invest. 1994; 94: 2392-6.

22. Mulvany MJ. Resistance vessel structure and function in the etiology of hypertension studied in F2-generation hypertensive-normotensive rats. J Hypertens. 1988; 6: 655-63.

23. Hofmann T, Obukhov AG, Schaefer M, et al. Direct activation of human TRPC6 and TRPC3 channels by diacylglycerol. Nature. 1999; 397: 259-63.

24. Lee YM, Kim BJ, Kim HJ, et al. TRPC5 as a candidate for the nonselective cation channel activated by muscarinic stimulation in murine stomach. Am J Physiol Gastrointest Liver Physiol. 2003; 284: G604-16.

25. Liu X, Bandyopadhyay BC, Singh BB, et al. Molecular analysis of a store-oper- ated and 2-acetyl-sn-glycerol-sensitive non-selective cation channel. Heteromeric assembly of TRPC1-TRPC3. J Biol Chem. 2005; 280: 21600-6.

26. Kiselyov K, Xu X, Mozhayeva G, et al. Functional interaction between InsP3 receptors and store-operated Htrp3 channels. Nature. 1998; 396: 478-82.

27. Trebak M, Bird GS, McKay RR, et al. Comparison of human TRPC3 channels in receptor-activated and store-operated modes. Differential sensitivity to channel blockers suggests fundamental differences in channel composition. J Biol Chem. 2002; 277: 21617-23.

28. Zagranichnaya TK, Wu X, Villereal ML. Endogenous TRPC1, TRPC3, and TRPC7 proteins combine to form native storeoperated channels in HEK-293 cells. $J$ Biol Chem. 2005; 280: 29559-69.

29. Yoshida J, Ishibashi T, Imaizumi N, et al. Capacitative $\mathrm{Ca}^{2+}$ entries and mRNA expression for TRPC1 and TRPC5 channels in human epidermoid carcinoma A431 cells. Eur J Pharmacol. 2005; 510: 217-22.

30. Rahman A, Hughes A, Matchkov V, et al. Antiphase oscillations of endothelium and smooth muscle $[\mathrm{Ca} 2+]$ in vasomotion of rat mesenteric small arteries. Cell Calcium. 2007; 42: 536-47.

31. Rose RA, Hatano N, Ohya S, et al. C-type natriuretic peptide activates a non-selective cation current in acutely isolated rat cardiac fibroblasts via natriuretic peptide $\mathrm{C}$ receptor-mediated signalling. J Physiol. 2007; 580: 255-74.

32. Baba A, Yasui T, Fujisawa $\mathrm{S}$, et al. Activity-evoked capacitative $\mathrm{Ca}^{2+}$ entry: implications in synaptic plasticity. J Neurosci. 2003; 23: 7737-41.

33. Zhu X, Jiang M, Birnbaumer L. Receptoractivated $\mathrm{Ca}^{2+}$ influx via human Trp3 stably expressed in human embryonic kidney (HEK)293 cells. Evidence for a non-capacitative $\mathrm{Ca}^{2+}$ entry. J Biol Chem. 1998; 273: 133-42.

34. Hajduczok G, Chapeau MW, Ferlic RJ, et al. Gadolinium inhibits mechanoelectrical transduction in rabbit carotid baroreceptors. J Clin Invest. 1994; 94: 2392-6.

35. Sada T, Koike H, Ikeda M, et al. Cytosolic free calcium of aorta in hypertensive rats. Chronic inhibition of angiotensin converting enzyme. Hypertension. 1990; 16: 245-51. 